\title{
AN EMBEDDING THEOREM FOR SPACES OF CONVEX SETS
}

\section{HANS RÅDSTRÖM}

1. Let $L$ be a real linear topological space. If $A$ and $B$ are convex sets and $\lambda$ is a real number, the sets $A+B$ and $\lambda A$ are defined and convex. Here $A+B=\{z \mid z=x+y, x \in A, y \in B\}, \lambda A=\{z \mid z=\lambda x$, $x \in A\}$. These operations satisfy

$$
\begin{gathered}
(A+B)+C=A+(B+C), \quad A+B=B+A, \\
\lambda(A+B)=\lambda A+\lambda B, \quad \lambda_{1}\left(\lambda_{2} A\right)=\left(\lambda_{1} \lambda_{2}\right) A, \quad 1 A=A,
\end{gathered}
$$

so that the set of all convex sets of $L$ is a commutative semigroup under addition. If the situation had been such that it was not only a semigroup but also a group, and if furthermore the second distributive law for multiplication with scalars had been true, then the set of convex sets would have been a vector space. The second distributive law: $\left(\lambda_{1}+\lambda_{2}\right) A=\lambda_{1} A+\lambda_{2} A$ is, however, true if $\lambda_{1}$ and $\lambda_{2}$ have the same sign, in particular if both are positive. It is therefore natural to ask whether the additive semigroup can be embedded in a group and whether multiplication with scalars can be extended to this group in such a way that the resulting system becomes a vector space, and so that for positive scalars the new multiplication coincides with the original one on the semigroup.

The object of this paper is to prove such an embedding theorem for the case in which $L$ is a normed linear space. We shall, however, not embed the class of all convex sets but only certain subclasses such as the class of all compact convex sets. Further we shall make the obtained vector space into a normed linear space by extending the Hausdorff distance $[3, \text { p. 146 }]^{1}$ between two convex sets. This is all done by using the well known classical method of extending commutative semigroups, which is used for example when defining negative numbers. The use of this method is made possible by the fact that the law of cancellation holds in our semigroup.

2. The following theorem contains all information we need acquire by use of the extension method.

THEOREM 1. A. Let $M$ be a commutative semigroup in which the law of cancellation holds. That is: For $A, B, C \in M$ we have $1 .(A+B)+C$

Received by the editors July 31, 1950 and, in revised form, April 1, 1951.

${ }^{1}$ Numbers in brackets refer to the references cited at the end of the paper. 
$=A+(B+C), 2 . A+B=B+A, 3 . A+C=B+C$ implies $A=B$. Then $M$ can be embedded in a group $N$. Furthermore $N$ can be chosen so as to be minimal in the following sense: If $G$ is any group in which $M$ is embedded, then $N$ is isomorphic to a subgroup of $G$ containing $M$.

B. If a multiplication by non-negative real scalars satisfying: 4. $\lambda(A+B)=\lambda A+\lambda B$, 5. $\left(\lambda_{1}+\lambda_{2}\right) A=\lambda_{1} A+\lambda_{2} A$, 6. $\lambda_{1}\left(\lambda_{2} A\right)=\lambda_{1} \lambda_{2} A$, 7. $1 A=A$ is defined on $M$, then a multiplication by real scalars can be defined on $N$ so as to make $N$ a vector space and so that for $\lambda \geqq 0$ and $A \in M$ the product $\lambda A$ coincides with the one given on $M$.

C. If further a metric $d(A, B)$ is given on $M$ and satisfying: 8 . $d(A+C, B+C)=d(A, B), 9 . d(\lambda A, \lambda B)=\lambda d(A, B), 10 . A+B$ and $\lambda A$ are continuous operations in the topology defined by $d$, then a metric can be defined on $N$ so as to make $N$ a normed linear space and so that if $A, B \in M$, the distance between $A$ and $B$ equals $d(A, B)$.

Proof. Once $N$ is defined, the necessary verifications of the axioms for groups, vector spaces, and normed spaces are entirely trivial and will not be carried through in detail.

The set $N$ shall consist of certain equivalence classes of pairs, $(A, B)$, of elements of $M$. The equivalence relation, denoted by " ," is defined by: $(A, B) \sim(C, D)$ if and only if $A+D=B+C$. The equivalence class containing the pair $(A, B)$ shall be denoted by $[A, B]$. In order to embed $M$ in $N$ we identify any element $A$ of $M$ with the class $[A+B, B]$.

Addition in $N$ is defined by $[A, B]+[C, D]=[A+C, B+D]$. If $\lambda$ is a non-negative scalar, we define $\lambda[A, B]=[\lambda A, \lambda B]$. If $\lambda$ is negative, we define $\lambda[A, B]=[|\lambda| B,|\lambda| A]$. The metric $\delta$ on $N$ is defined by $\delta([A, B],[C, D])=d(A+D, B+C)$.

After having made these definitions we should verify that they really determine the corresponding functions uniquely. As an example we choose the metric. Suppose that $[A, B]=\left[A_{1}, B_{1}\right]$ and $[C, D]=\left[C_{1}, D_{1}\right]$. Thus $\delta=\delta([A, B],[C, D])=d(A+D, B+C)$. Because of 8 , we may add $A_{1}+B_{1}+C_{1}+D_{1}$ and get $\delta=d\left(\left(A+B_{1}\right)\right.$ $\left.+\left(C_{1}+D\right)+A_{1}+D_{1},\left(A_{1}+B\right)+\left(C+D_{1}\right)+B_{1}+C_{1}\right)$. Since $A+B_{1}=A_{1}$ $+B$ and $C+D_{1}=C_{1}+D$, we obtain $\delta=d\left(A_{1}+D_{1}, B_{1}+C_{1}\right)=\delta\left(\left[A_{1}, B_{1}\right]\right.$, $\left.\left[C_{1}, D_{1}\right]\right)$.

A detailed discussion of part $\mathrm{A}$ of the theorem may be found in Graves [2, p. 24]. The verifications necessary for part B are easy although somewhat lengthy. As an example we choose to prove the second distributive law. The proof requires discussion of a number of cases. As the argument is essentially the same in the different cases, we do only one typical case. Suppose $\lambda_{1}>0, \lambda_{2}<0, \lambda_{1}+\lambda_{2}<0$. Thus $\left(\lambda_{1}+\lambda_{2}\right)[A, \quad B]=\left[\begin{array}{ll}\left(-\lambda_{1}-\lambda_{2}\right) B, & \left(-\lambda_{1}-\lambda_{2}\right) A\end{array}\right]=\left[\left(-\lambda_{1}-\lambda_{2}\right) B\right.$ 
$\left.+\lambda_{1}(A+B), \quad\left(-\lambda_{1}-\lambda_{2}\right) A+\lambda_{1}(A+B)\right]=\left[-\lambda_{2} B+\lambda_{1} A, \quad-\lambda_{2} A+\lambda_{1} B\right]$ $=\left[-\lambda_{2} B,-\lambda_{2} A\right]+\left[\lambda_{1} A, \lambda_{1} B\right]=\lambda_{2}[A, B]+\lambda_{1}[A, B]$. This proves the second distributive law.

Concerning part $\mathrm{C}$ we have to verify that $\delta$ is a metric on $N$, that it is homogeneous and invariant under translations in $N$, and that it makes addition and multiplication continuous. Let $x=[A, B] \in N$ and $y=[C, D] \in N$. Suppose $\delta(x, y)=0$. Thus $d(A+D, B+C)=0$ or $A+D=B+C$ which means $(A, B) \sim(C, D)$ or $x=y$. Conversely $x=y$ implies $\delta(x, y)=0$. The law $\delta(x, y)=\delta(y, x)$ is immediate. To prove the triangular inequality, put $z=[E, F]$. Thus $\delta(x, \quad z)=d(A+F, \quad B+E)=d((A+D)+(C+F), \quad(B+C)+(D+E))$ $\leqq d((A+D)+(C+F),(B+C)+(C+F))+d((B+C)+(C+F),(B+C)$ $+(D+E))=d(A+D, B+C)+d(C+F, D+E)=\delta(x, y)+\delta(y, z)$. The rest of the verification is easily done.

At last we notice that $\delta(x, y)$, because of the invariance under translations, is really a function of only one variable, namely $x-y$ $(=x+(-1) y)$, so that we can put $d(x, y)=\|x-y\|$. The function $\|x\|$ satisfies the axioms for a norm, which proves part $\mathrm{C}$.

3. We now proceed to prove that the law of cancellation holds for certain classes of convex sets.

Lemma 1. Let $A, B$, and $X$ be given sets in a real normed linear space Suppose $B$ closed and convex, $X$ bounded, and that $A+X \subset B+X$. Then $A \subset B$.

Proof. Let $a$ be any element of $A$. We shall show that it has to be an element of $B$. We know that given any $x_{1} \in X$ we have $a+x_{1}$ $\in B+X$, that is, there exist $b_{1} \in B$ and $x_{2} \in X$ with $a+x_{1}=b_{1}+x_{2}$. For the same reason $b_{2} \in B$ and $x_{3} \in X$ with $a+x_{2}=b_{2}+x_{3}$ must exist. Repeat the procedure indefinitely and sum the first $n$ of the equations obtained. We get: $n a+\sum_{i=1}^{n} x_{i}=\sum_{i=1}^{n} b_{i}+\sum_{i=2}^{n+1} x_{i}$ or $n a+x_{1}$ $=\sum_{i=1}^{n} b_{i}+x_{n+1}$ or $a=(1 / n) \sum_{i=1}^{n} b_{i}+x_{n+1} / n-x_{1} / n$. Put $(1 / n) \sum_{i=1}^{n} b_{i}$ $=c_{n}$. Thus $a=c_{n}+x_{n+1} / n-x_{1} / n$. We observe that $c_{n} \in B$ for all $n$ because of the convexity of $B$, that $x_{1} / n$ tends to the origin and that $x_{n+1} / n$ tends to the origin because of the boundedness of $X$. Therefore $c_{n}$ converges to $a$. But, $B$ being closed, this implies that $a \in B$.

Lemma 2. If $A$ and $B$ are closed, convex sets in a real normed linear space and $X$ is bounded, then $A+X=B+X$ implies $A=B$.

4. The next lemma deals with the invariance of Hausdorff distance.

LEMMA 3. Let $A$ and $B$ be convex sets in a real normed linear space. Suppose also that $A+\lambda S$ and $B+\lambda S$ are closed for all $\lambda \geqq 0$, where $S$ is 
the unit sphere. Let $X$ be any bounded set. Then $d(A, B)=d(A+X$, $B+X)$.

Proof. 'Let $S$ denote the unit sphere of the space. Consider the following four inequalities
(1) $A+\lambda S \supset B$,
(2) $B+\lambda S \supset A$,
(3) $A+X+\lambda S \supset B+X$, and
(4) $B+X+\lambda S \supset A+X$.

Put $d_{1}=d(A, B)$ and $d_{2}=d(A+X, B+X)$. Then $d_{1}$ equals the infimum of the positive numbers $\lambda$ for which (1) and (2) hold. Similarly for $d_{2}$ and (3) and (4). Since (3) and (4) follow from (1) and (2) respectively by adding $X$, we have $d_{1} \geqq d_{2}$. Conversely since, by Lemma 1 , cancelling $X$ is allowed in (3) and (4), we obtain $d_{1} \leqq d_{2}$, which proves Lemma 3.

5. Combining the results obtained, we easily prove the following embedding theorem.

THEOREM 2. Let $L$ be a real normed linear space and $M$ any space the points of which are closed, bounded convex sets in $L$, and which has the following properties:

1. $M$ is closed under addition and multiplication by non-negative scalars.

2. If $A \in M$ and $S$ is the unit sphere of $L$, then $A+S$ is closed.

3. $M$ is metrized by the Hausdorff metric.

Then $M$ can be embedded as a convex cone in a real normed linear space $N$ in such a way that

a. the embedding is isometric,

b. addition in $N$ induces addition in $M$,

c. multiplication by non-negative scalars in $N$ induces the corresponding operation in $M$.

Furthermore, $N$ can be chosen so as to be minimal in the following sense: If $H$ is any real normed linear space in which $M$ is embedded in the above fashion, then $H$ contains a subspace containing $M$ and isomorphic to $N$.

The following three examples satisfy the conditions put on $M$.

$\alpha$. The set of all finite-dimensional compact convex sets.

$\beta$. The set of all compact convex sets.

$\gamma$. The set of all closed, bounded and regularly convex sets, provided $L$ is an adjoint space. If $L$ is a reflexive space the set of all closed, bounded, convex sets will therefore satisfy the assumptions.

Proof. Using Theorem 1 we have to verify the conditions 1-10, all of which except 3 and 8 are well known facts from the theory of 
convex sets. Condition 3 is supplied by Lemma 2 and 8 by Lemma 3 . In order to see that the sets $\alpha, \beta$, and $\gamma$ can be used for $M$ we have to verify that $A \in M$ implies $A+S$ closed which is easy for $M=\alpha$ and $\beta$. For the case $M=\gamma$ see Krein and Smulian [5].

6. The idea of embedding spaces of convex sets in linear spaces seems to go back to Brunn [1]. For an interesting application of this idea and further references see Inzinger [4].

I am indebted to the referee for pointing out the applicability of Theorem 2 to the set $\gamma$ and for some other valuable remarks.

\section{REFERENCES}

1. H. Brunn, Ueber Curven ohne Wendepunkte, Munich, 1889.

2. L. Graves, Theory of functions of real variables, New York and London, 1946.

3. F. Hausdorff, Mengenlehre, Berlin and Leipzig, 1927.

4. R. Inzinger, Stiltzbare Bereiche, trigonometrische Polynome und Defizite höherer Ordnung, Monatshefte für Mathematik vol. 53 (1949) p. 302.

5. V. F. Krein and V. Smulian, On regularly convex sets in the space conjugate to a Banach space, Ann. of Math. vol. 41 (1940) p. 556.

UNIVERSITY OF STOCKHOLM 